\title{
Surface Wave Tomography
}

\section{Don L. Anderson}

Seismological Laboratory, California Institute of Technology, Pasadena, CA 91125

Surface waves are now being used by several groups to map lateral heterogeneity [ $\mathrm{Na}$ kanishi and Anderson, 1982, 1983, 1984a, b; Woodhouse and Dziewonski, 1984] and anisotropy [Tanimoto and Anderson, 1984a, $b$; Nataf et al. 1984] of the upper mantle on a global basis. The method involves measuring the phase and/or group velocity over hundreds of small arcs and long arcs connecting earthquakes and seismic stations. These averages are then converted to three-dimensional images of the seismic velocity structure, and, hence, this is a form of romography. The large amount of data processing required is made feasible by the existance of long-period digital seismic networks including IDA (International Deployment of Accelerometers), SRO (Seismic Research Observatories), and GDSN (Global Digital Seismic Network). These instruments are operated by a variety of university and government groups including University of California, San Diego, U.S. Geological Survey, D.A.R.P.A., and U.S. Department of Energy with the cooperation of many countries. The global coverage is still very sparse compared to the analog W.W.S.S.N. (World Wide Standardized Seismic Network), but preliminary results are very encouraging. The possibility of an expanded global digital network of broadband seismic stations is now being pursued actively by the United States and several other countries. Because of the sparseness of the present network, mantle structure can only be mapped with fairly low resolving power. Only features with half wavelength of the order of $2,000 \mathrm{~km}$ can be detected.

The maps on the cover show the results from one recent study [Nataf et al., 1984]. Shown are the parameters VSV and XI at two depths, 250 and $350 \mathrm{~km}$. VSV is the velocity of vertically polarized shear waves, determined primarily from fundamental mode Rayleigh waves, and XI is related to the difference between VSH, the velocity of horizontally polarized shear waves, and VSV. The parameter XI is therefore a measure of anisotropy. Positive XI means VSH > VSV. Aggregates composed of $a$ axis horizontal olivine crystals, for example, are expected to have XI $>0$. This situation is probably diagnostic of horizontal flow. XI $<0$ is expected for vertical $a$ axis orientation of olivine and implies vertical flow. Blue areas in the maps represent fast regions or VSH > VSV. Orange areas are slower than average or VSH $<$ VSV. The maps are spherical harmonic representations including coefficients up to and including order and degree 6.

In spite of the lack of short wavelength information, there is much important information in these maps. Mid-oceanic ridges and regions of recent volcanic activity are generally slow at $250 \mathrm{~km}$. The regions near the Tasman Sea, New Zealand; Red Sea, African rift; and western North America are slow. The central Pacific, between Hawaii and Tahiti, is also slow at this depth. Fast regions include the Canadian and Fennoscandian shields and the Siberian platform, as expected, but also the North Pacific and the eastern Indian Ocean. Many hot spots are on the edges of low velocity regions rather than centrally located.

The parameter XI is negative over regions of upwelling (East Pacific Rise, Antarctic-Pacific Rise, South Indian Rises, Mid-Atlantic Rise, and the Red Sea region) and areas of presumed downwelling (Japan, Philippines, Mariannas, Sumatra). Plate interiors are gen- erally positive XI, suggesting horizontal flow at $250 \mathrm{~km}$

The maps at $350 \mathrm{~km}$ are similar, as they should be since a high degree of correlation between 220 and $400 \mathrm{~km}$ was assumed in the inversion. The differences are therefore particularly instructive. The central Pacific, Red Sea, and mid-Atlantic ridge slow anomalies persist, suggesting that these are relatively deep seated. Most shields are no longer evident. The thermal anomaly associated with the East Pacific Rise appears to be displaced. At $450 \mathrm{~km}$ depth, not shown, most ridges are fast and most subduction regions are also fast. The velocity and XI parameter at 350 $\mathbf{k m}$ are consistent with upwelling flow along the East Pacific Rise, the central and southern mid-Atlantic Rise, and the Red Sea area. These parameters are consistent with downwelling in the western Pacific. An upwelling is implied in the south central Pacific.

\section{Acknowledgments}

This research was supported by National Science Foundation grant EAR81-15236 and National Aeronautics and Space Administration contract NSG-7610. Contribution number 4039, Division of Geological and Planetary Sciences, California Institute of Technology, Pasadena, California 91125.

\section{References}

Nakanishi, I., and D. L. Anderson, Worldwide distribution of group velocity of mantle Rayleigh waves as determined by spherical harmonic inversion, Bull. Seismol. Soc. $A m ., 72,1185-1194,1982$.

Nakanishi, I., and D. L. Anderson, Measurement of mantle wave velocities and inversion for lateral heterogeneity and anisotropy, 1,J. Geophys. Res., 88, 10,267-10,283, 1983.

Nakanishi, I., and D. L. Anderson, Aspherical heterogeneity of the mantle from phase velocities of mantle waves, Nature, 307 , 117-121, 1984a.

Nakanishi, I., and D. L. Anderson, Measurements of mantle velocities and inversion for lateral heterogeneity and anisotropy, II, Analysis by the single-station method, Geophys, J. R. Astron. Soc., in press, $1984 b$.
Cover. The velocity of vertically polarized shear waves, VSV, and inisotropy, XI, at 250 and $350 \mathrm{~km}$ depth derived from surface wave tomographic inversion of global Love and Rayleigh wave dispersion from model of Nataf et al. [1984]. (Figure courtesy of Don L. Anderson. See Anderson article this issue for complete description.) 